Research Paper

\title{
Innate immunity recovers earlier than acquired immu- nity during severe postoperative immunosuppression
}

\author{
Gunnar Lachmann, Clarissa von Haefen, Johannes Kurth, Fatima Yuerek, Claudia Spies ${ }^{\bowtie}$ \\ Department of Anesthesiology and Operative Intensive Care Medicine, Campus Charité Mitte and Campus Virchow-Klinikum, Charité - Universitätsmedizin \\ Berlin, Germany. \\ $\triangle$ Corresponding author: Claudia Spies, Professor of Anesthesiology and Intensive Care Medicine, Department of Anesthesiology and Operative Intensive Care \\ Medicine, Campus Charité Mitte and Campus Virchow-Klinikum, Charité - Universitätsmedizin Berlin. Augustenburger Platz 1, D-13353 Berlin, Germany. \\ Phone: +4930450551001 Fax:+4930450551909 Email: claudia.spies@charite.de \\ (C) Ivyspring International Publisher. This is an open access article distributed under the terms of the Creative Commons Attribution (CC BY-NC) license \\ (https://creativecommons.org/licenses/by-nc/4.0/). See http://ivyspring.com/terms for full terms and conditions.
}

Received: 2017.06.12; Accepted: 2017.09.10; Published: 2018.01.01

\begin{abstract}
Background: Postoperative immune suppression, particularly a loss of cell-mediated immunity, is commonly seen after surgery and is associated with worse outcome, i.e. delayed wound healing, infections, sepsis, multiple-organ failure and cancer recurrence. However, the recovery of immune cells focusing on differences between innate and acquired immunity during severe postoperative immunosuppression is not investigated. Methods: In this retrospective randomized controlled trial (RCT) subgroup analysis, 10 postoperatively immune suppressed patients after esophageal or pancreatic resection were analyzed. Innate and acquired immune cells, the expression of human leukocyte antigen-D related on monocytes (mHLA-DR), lipopolysaccharide (LPS)-induced monocytic TNF- $\alpha$ and IL-10 secretion ex vivo, Concanavalin A (Con A)-induced IFN- $-\gamma, T N F-\alpha$, IL-2, IL-4, IL-5 and IL-10 release were measured preoperatively (od) until day 5 after surgery (pod5). Recovery of immune cells was defined by a significant decrease respectively increase after a significant postoperative alteration. Statistical analyses were performed using nonparametric statistical procedures. Results: Postoperative alterations of innate immune cells recovered on pod2 (eosinophils), pod3 (neutrophils) and pod5 (mHLA-DR, monocytic TNF- $\alpha$ and IL-10 secretion), whereas alterations of acquired immune cells (lymphocytes, $T$ cells, $T$ helper cells, and cytotoxic $T$ cells) did not recover until pod5. Peripheral blood T cells showed an impaired production of the $T$ helper (Th) 1 cytokine IFN-Y upon Con A stimulation on pod I, while Th2 specific cytokine release did not change until pod5. Conclusions: Innate immunity recovered earlier than acquired immunity during severe postoperative immunosuppression. Furthermore, we found a more anti- than pro-inflammatory $\mathrm{T}$ cell function on the first day after surgery, while $\mathrm{T}$ cell counts decreased.
\end{abstract}

Key words: immune suppression; monocytic function; T cell function; innate immunity; acquired immunity

\section{Introduction}

Postoperative immune suppression particularly a loss of cell-mediated immunity is commonly seen after surgery due to an increased release of immune suppressing hormones such as catecholamines, prostaglandins and cortisol depending on the amount of surgical stress and tissue damage [1,2]. Blood transfusion, hypothermia, dehydration and anesthetics can further attenuate immunity [3-6]. An impaired immunity after surgery is associated with worse outcome, i.e. delayed wound healing, infections, sepsis, multiple-organ failure and cancer recurrence [1, 7-12]. In particular, postoperative immunosuppression comprises decreased numbers of natural killer (NK) cells, T lymphocytes, as well as an impaired function of $\mathrm{T}$ lymphocytes and monocytes including a suppressed expression of human leukocyte antigen-D related on monocytes (mHLA-DR) [1, 13-19]. B lymphocytes seem to be less effected $[1,20]$. Furthermore, increasing numbers of $T$ regulatory (Treg) cells and neutrophils often occur 
after surgery [13, 21]. While major surgery may suppress cellular immunity for several days, humoral immunity remains relatively intact [8]. However, the recovery of immune cells focusing on differences between innate and acquired immunity during severe postoperative immunosuppression has not been investigated, yet.

\section{Patients and Methods}

\section{Study Participants and Treatment}

This retrospective subgroup analysis of a previously published study of our research group [22, 23] investigated innate and acquired immune cells as well as monocytic and $\mathrm{T}$ cell immune function during severe postoperative immune suppression (mHLA-DR $\leq 10,000$ antigens per cell on pod1) that were measured in 10 out of 20 patients of the placebo group of the bigger cohort until pod5 after elective esophageal or pancreatic resection (measurement had to be stopped after 10 patients for economic reasons, no selection of the patients; Figure 1). All patients received guideline-based anesthesiological and surgical treatment according to our standard operating procedures [24].

\section{Measurement of parameters of immune function}

Blood samples were drawn from od until pod5. mHLA-DR and further parameters of immune function were measured from od until pod5. Expression of mHLA-DR was determined by cytometric analysis using a highly standardized quantitative assay as described earlier [25]. For determination of soluble mediators, ethylene diamine tetraacetic acid (EDTA) and heparin plasma samples were collected and stored at $-80^{\circ} \mathrm{C}$ until assay. All immunological parameters were analyzed in collaboration with the Institute of Medical Immunology and Berlin-Brandenburg Center for Regenerative Therapies (BCRT), Charité Universitätsmedizin Berlin, Berlin, Germany. White blood cell differential count was measured in a standard hematology analyzer (Sysmex). For flow cytometry analysis, lymphocyte subpopulations were identified using the following antibody combinations: CD45 for leukocytes, CD3+ for $\mathrm{T}$ lymphocytes, $\mathrm{CD} 3+\mathrm{CD} 4+$ for $\mathrm{T}$ helper cells (Th), CD3+CD8+ for cytotoxic T cells, CD2+CD3-CD16+ for natural killer (NK) cells and CD19+ for B lymphocytes. Cell phenotyping was performed by flow cytometry/fluorescence-activated cell sorting (FACS) on a FACSCalibur ${ }^{\mathrm{TM}}$ using CELLQuest ${ }^{\mathrm{TM}}$ Software (BD Biosciences). LPS-induced monocytic tumor necrosis factor alpha (TNF-a) and Interleukin (IL)-10 secretion ex vivo as well as Concanavalin A (Con A)-induced interferon gamma (IFN- $\gamma$ ), TNF- $\alpha$, IL-2, IL-4, IL-5 and IL-10 release were determined as described earlier [23].

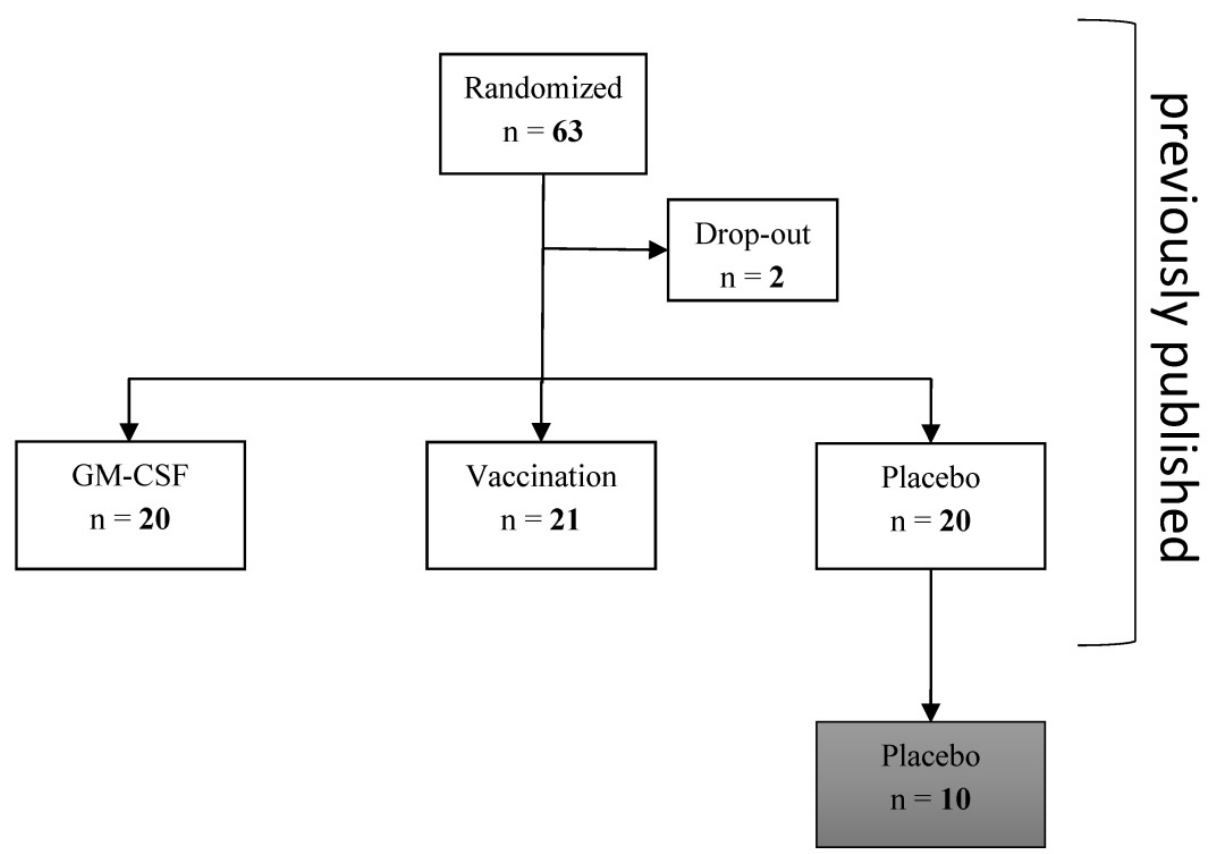

Figure 1. Consort diagram. 10 patients of the placebo group were analyzed in this subgroup analyses: immune cells and functional parameters were determined in these patients without any prior selection. 


\section{Statistical analysis}

Data were expressed according to their scaling as arithmetic mean \pm standard deviation (SD), median [25\%, 75\% quartiles], or frequencies [\%]. After exploratory data analysis, all tests were accomplished by means of non-parametric exact statistical tests. Longitudinal data were analyzed using nonparametric univariate procedures. For each immune parameter, we first tested over all six time points (od until pod5). In a second step, we determined the first significant postoperative alteration by comparing postoperative and presurgical values. The specific time point was then compared with further time points to determine the recovery of immune parameters, i.e. a significant decrease respectively increase after a significant postoperative alteration. A two-tailed p-value $<0.05$ was considered statistically significant. All tests should be understood as constituting exploratory data analysis, so that no adjustments for multiple testing have been made. Numerical calculations were performed using IBM@ SPSS(C) Statistics, Version 23.

\section{Results}

\section{Study population}

Basic patient characteristics, intraoperative- and outcome parameters are shown in Table 1. Some of the results were already shown to analyze group differences after postoperative immune stimulation [23].

\section{Innate immune cells}

Neutrophils showed significant differences from od until pod5 ( $\mathrm{p}<0.001$; Figure 2A), increased on pod1 $(\mathrm{p}=0.005)$ and recovered on pod3 $(\mathrm{p}=0.037)$. Eosinophils differed from od until pod5 $(\mathrm{p}=0.002$; Figure 2B), decreased on pod1 $(\mathrm{p}=0.018)$ and recovered on pod2 $(\mathrm{p}=0.043)$. Basophils differed from od until pod5 ( $\mathrm{p}=0.035$; Figure $2 \mathrm{C})$ and decreased on pod3 $(\mathrm{p}=0.047)$. NK cell counts showed significant differences from od until pod5 ( $p<0.001$; Figure 2D), decreased on $\operatorname{pod} 2(\mathrm{p}=0.024)$ and again on $\operatorname{pod} 3(\mathrm{p}=$ 0.015). Monocytes differed from od until pod5 ( $\mathrm{p}<$ 0.001; Figure 2E), increased on pod1 $(\mathrm{p}=0.007)$ and again on pod5 $(\mathrm{p}=0.050)$. mHLA-DR showed significant differences from od until pod5 $(\mathrm{p}<0.001$; Figure $2 \mathrm{~F})$, decreased on pod1 $(\mathrm{p}=0.005)$ and recovered on $\operatorname{pod} 5(\mathrm{p}=0.008)$.

\section{Function of innate immune cells}

TNF-a release of LPS-stimulated monocytes significantly decreased on $\operatorname{pod} 2(\mathrm{p}=0.038$; Figure $3 \mathrm{~A})$ and recovered on pod5 $(\mathrm{p}=0.050)$. IL-10 release of
LPS-stimulated monocytes decreased on $\operatorname{pod} 3(\mathrm{p}=$ 0.038 ; Figure $3 \mathrm{~B})$ and recovered on pod5 $(\mathrm{p}=0.012)$.

Table 1. Basic patient characteristics, intraoperative and outcome parameters.

\begin{tabular}{ll}
\hline & Placebo group (n = 10) \\
\hline Age [years] & $62(55-69)$ \\
Gender male/female [n] & $7 / 3$ \\
Body Mass Index [kg/m²] & $25.5(24.2-27.5)$ \\
Pancreatic/esophageal resection [n] & $6 / 4$ \\
ASA score II/III [n] & $7 / 3$ \\
Smokers/non-smokers [n] & $4 / 6$ \\
AUDIT score & $3(0-6)$ \\
Non-diabetes/diabetes [n] & $9 / 1$ \\
Metabolic equivalent (MET) <4/4-10/>10 & $0 / 8 / 2$ \\
Surgical time [min] & $308(280-378)$ \\
Intraop. blood loss [mL] & $600(313-950)$ \\
Intraop. mean blood glucose [mg/dL] & $127(122-142)$ \\
Intraop. max. blood lactate [mmol/L] & $1.0(0.8-1.3)$ \\
Intraop. mean systolic blood pressure [mmHg] & $113(109-117)$ \\
APACHE II score on admission to ICU & $12(9-16)$ \\
SAPS II score on admission to ICU & $22(12-27)$ \\
SOFA score on admission to ICU & $2(1-4)$ \\
TISS 28 score on admission to ICU & $32(27-36)$ \\
ICU stay [d] & $3.2(2.4-4.9)$ \\
Hospital stay [d] & $14.4(11.5-20.6)$ \\
Survived/deceased [n] & $10 / 0$ \\
\hline
\end{tabular}

Continuous quantities in median (25\%-75\% percentiles), frequencies with $\mathrm{n}(\%)$. ASA, American Society of Anesthesiologists; AUDIT score, Alcohol Use Disorders Identification Test; APACHE, Acute Physiology and Chronic Health Evaluation; SAPS, Simplified Acute Physiology Score; SOFA, Sequential Organ Failure

Assessment; TISS, Therapeutic Intervention Scoring System; ICU, Intensive Care Unit.

\section{Acquired immune cells und subsets}

Lymphocytes showed significant differences from od until pod5 ( $\mathrm{p}=0.007$; Figure $4 \mathrm{~A})$ and decreased on pod1 $(p=0.015)$. B cells did not show any significant differences (Figure 4B). $T$ cells showed significant differences from od until pod5 $(\mathrm{p}=0.027$; Figure $4 \mathrm{C})$ and decreased on pod1 $(\mathrm{p}=0.011)$. T helper cells decreased on pod1 $(p=0.007)$. Cytotoxic T cells showed significant differences from od until pod5 $(\mathrm{p}=$ 0.016 ; Figure 4E) and decreased on pod $2(p=0.005)$. The ratio of $\mathrm{T}$ helper and cytotoxic $\mathrm{T}$ cells significantly increased on pod3 $(\mathrm{p}=0.011$; Figure $4 \mathrm{~F})$ compared to pod1.

\section{Function of acquired immune cells}

After stimulation of whole-blood cultures for 24 $\mathrm{h}$ with Con A, the cytokines for Th1 and Th2 responsiveness IFN- $\gamma$, TNF- $\alpha$, IL-2, IL-4, IL-5 and IL-10 were measured. While the Th1 cytokine IFN- $\gamma$ decreased on pod1 $(p=0.028$; Figure $5 \mathrm{~A})$, TNF- $\alpha$ and IL-2 did not change significantly after surgery (Figure 5B, C). The Th2 cytokines IL-4, IL-5 and IL-10 did not show any differences after surgery (Figure 5D, E, F). 


\section{Discussion}

The major finding of this subgroup analysis is that innate immunity recovered earlier than acquired immunity during severe postoperative immunesuppression. To the best of our knowledge, no other

\section{A Neutrophils}

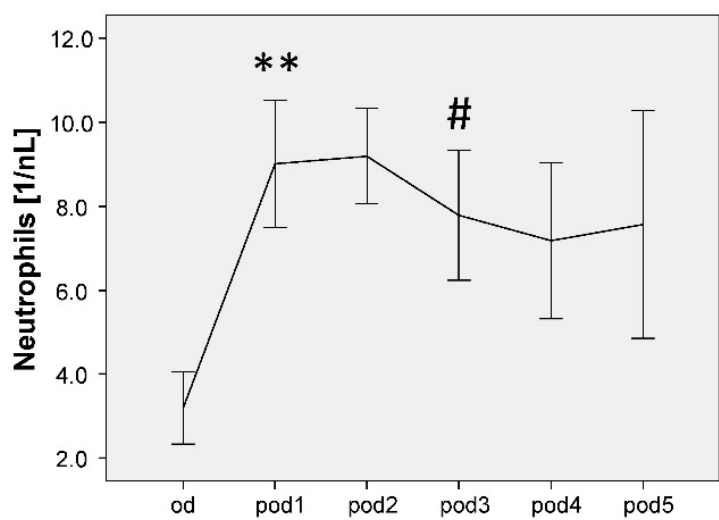

C

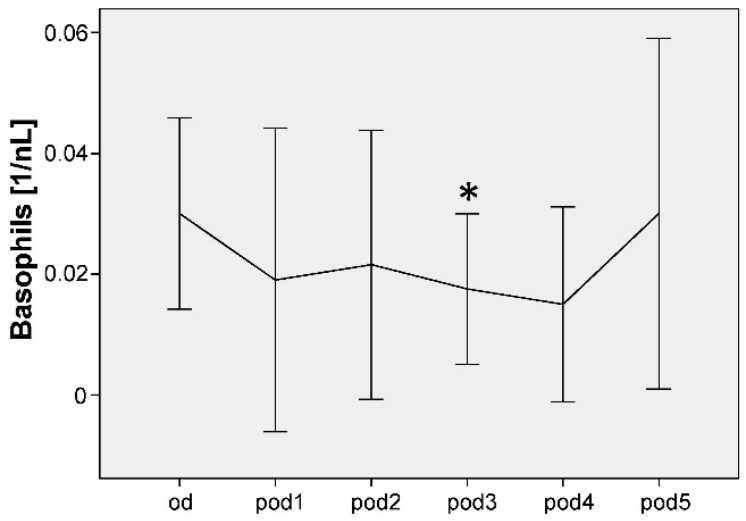

E Monocytes

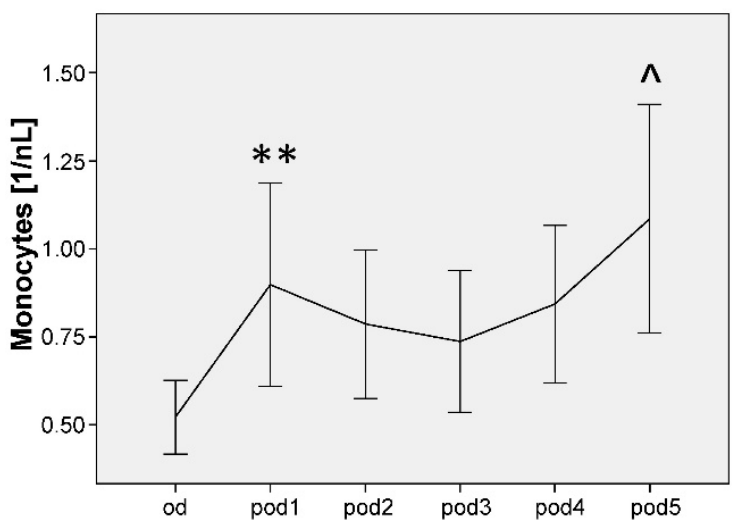

study has investigated differences in recovery between innate and acquired immune cells during severe postoperative immunosuppression after major cancer surgery, yet.

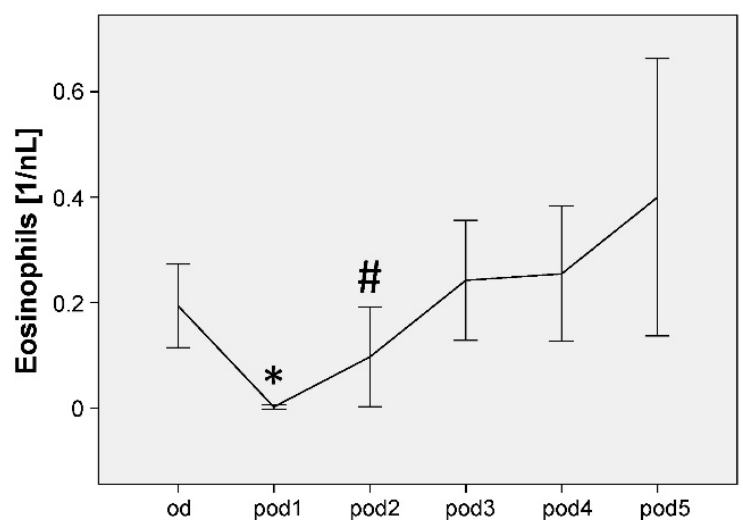

D

NK cells

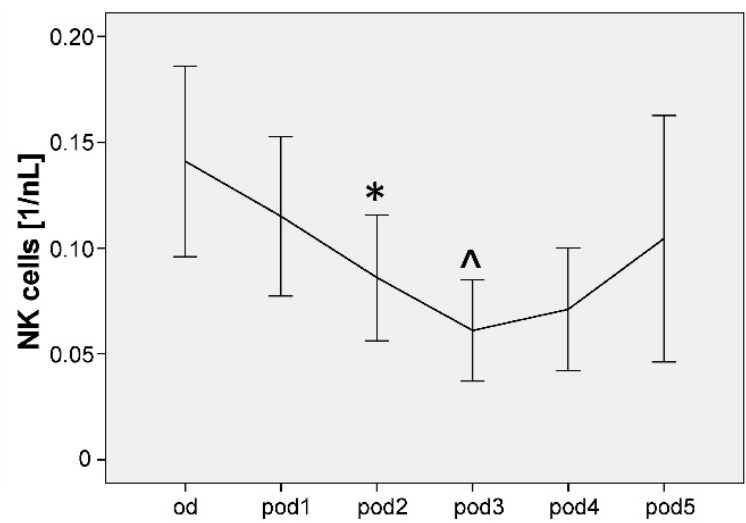

F $\quad$ MHLA-DR

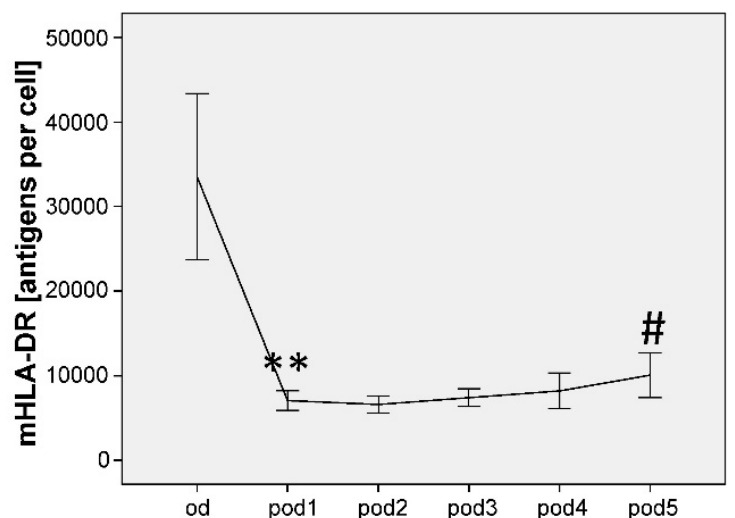

Figure 2. Neutrophils, eosinophils, basophils, natural killer (NK) cells, monocytes and mHLA-DR from day of surgery before surgery (od) until day 5 after surgery (pod5). Neutrophils increased on podl and recovered on pod3, eosinophils decreased on podl and recovered on pod2, basophils decreased on pod3, NK cells decreased on pod2 and again on pod3 compared to pod2. Monocytes increased on pod I and again on pod5 compared to pod I. mHLA-DR decreased on pod I and recovered on pod5. **P $<0.01$, *P $<0.05$ represent the first significant differences between pre- and postsurgical values. ${ }^{\wedge} \mathrm{P}<0.05$ represents the second significant alteration compared to the prior significant alteration. \#P<0.05 represents recovery, i.e. the first significant in- respectively decrease after significant postoperative alteration. Error bars with $95 \%$ confidence intervals. 
A

TNF- $\alpha$ after LPS

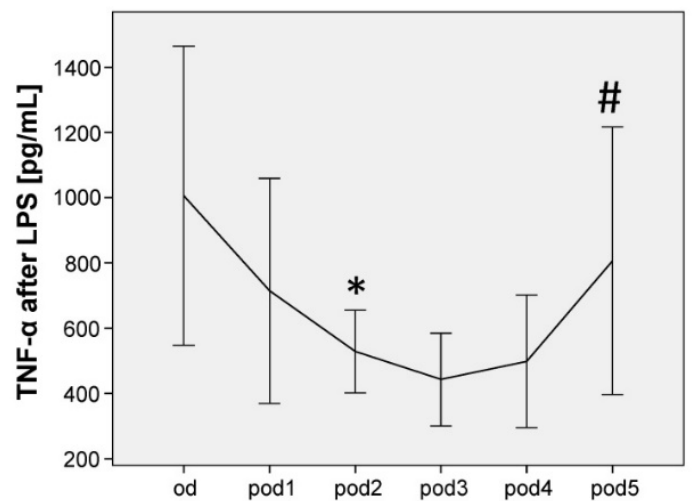

B

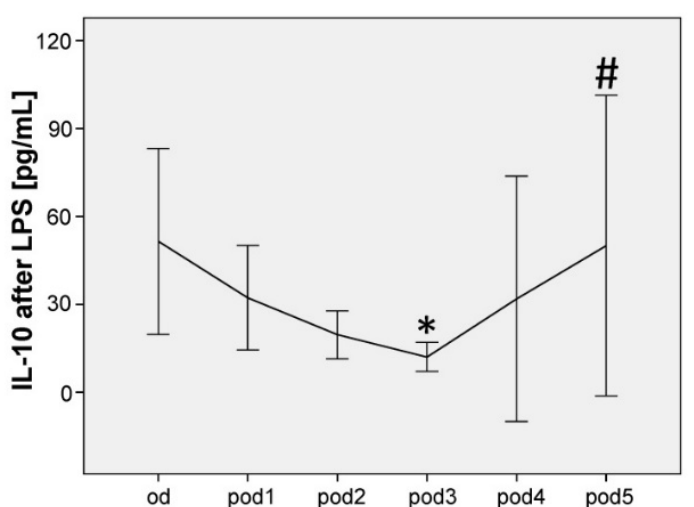

Figure 3. TNF- $\alpha$ and IL-10 release of LPS-stimulated monocytes from day of surgery before surgery (od) until day 5 after surgery (pod5). TNF- $\alpha$ decreased on pod 2 and recovered on pod5, IL- 10 decreased on pod3 and recovered on pod5. $* \mathrm{P}<0.05$ represents the first significant differences between pre- and postsurgical values. \#P<0.05 represents recovery, i.e. the first significant in- respectively decrease after significant postoperative alteration. Error bars with $95 \%$ confidence intervals.

In general, innate and acquired immune defense play a key role in eliminating of infective pathogens and malignancies. When stimulated by pathogens, immune cells of the innate immune system produce cytokines and other co-stimulatory molecules, whereas the adaptive immune system is essential for immunologic memory and release of antibodies for more specific immune responses [26]. Interactions between innate and acquired immunity, i.e. monocytes and $\mathrm{T}$ lymphocytes with antigen presentation and consequent $\mathrm{T}$ cell response are essential for adequate immune function [27]. Particularly in postsurgical patients, profound immune alterations occur with a highly attenuated and restricted immunity [1], which can be measured by a decreased mHLA-DR [12].

We included immune suppressed patients with a mHLA-DR concentration not higher than 10,000 antibodies per monocyte on day one after surgery, which indicates a highly suppressed immune function. Therefore, all patients showed a postoperative immune suppressed state: counts of basophils, eosinophils, NK cells, lymphocytes except of $\mathrm{B}$ cells, as well as function of monocytes (mHLA-DR, TNF- $\alpha$ and IL-10 release of LPS-stimulated monocytes) and T cells (IFN- $\gamma$ release after stimulation) decreased, whereas counts of neutrophils and monocytes increased. Immune alterations during the postoperative period are well described and in accordance with our findings $[1$, 13-19].

The exact pathological mechanism for an impaired postoperative immune function is still speculative. Perioperatively secreted catecholamines and prostaglandins are assumed to be a major cause of postoperative immune suppression following anesthesia [2, 6]. Latest research suggests that so-called alarmins released depending on tissue damage might lead to a pronounced pro-inflammatory response [28]. The initial pro-inflammatory response aims to activate immunity to the site of injury and induces a systemic anti-inflammatory state whose physiological effect should prevent the formation of inflammatory tissue and organ damage with the negative effect of leading to a pronounced postoperative immunosuppression [29].

We found an earlier recovery of innate immune cells compared to acquire immune cells. Concretely, innate immune cells recovered on pod2 (eosinophils), pod3 (neutrophils) and pod5 (monocytic function), whereas counts of acquired immune cells (lymphocytes, T cells, T helper and cytotoxic T cells) did not recover until pod5. B cells did not decrease postoperatively. In the present study, we additionally investigated changes in lymphocyte subsets in patients with postoperative immunosuppression. Our findings suggest that the homeostasis of $\mathrm{T}$ cells is perturbed in immune suppressive patients after surgery. The decreased numbers of $\mathrm{T}$ cells in peripheral blood of immunosuppressed patients may reflect an increased rate of apoptosis of these cells. Clinical research showed an influence of surgical procedures on circulating blood lymphocyte apoptosis [30]. Considering functional parameters of T cells, Th1 specific cytokines were decreased and Th2 specific cytokines were unchanged on day one after surgery, which suggests an anti-inflammatory state immediately postoperatively. The imbalance of Th1 and Th2 cytokines is associated with an increased susceptibility to postoperative infections [31]. Our results therefore suggest that particularly acquired immune cells are highly vulnerable to postoperative immunosuppression, and compared to innate immune cells remain suppressed for a longer period of time. 
It is of major importance to minimize postoperative immunosuppression due to its high impact on outcome regarding sepsis and cancer recurrence $[8,9,11]$. Adequate perioperative pain control particularly epidural analgesia was shown to reduce postoperative immune suppression after major abdominal surgery [32]. Furthermore, perioperative

A Lymphocytes
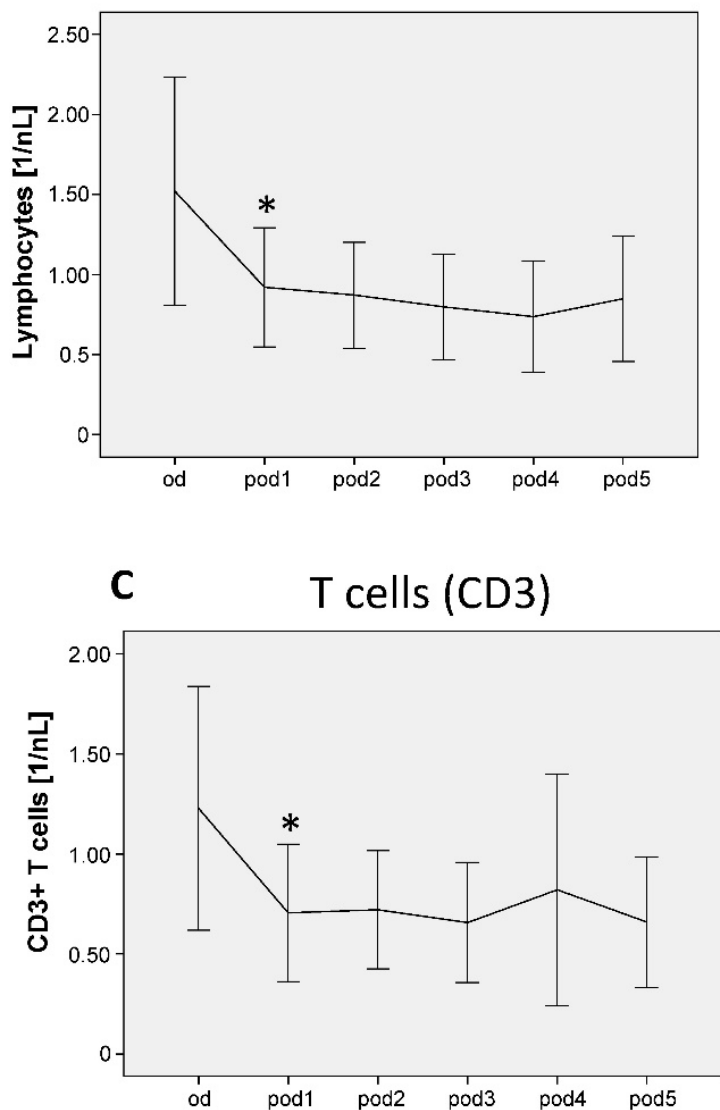

E Cytotoxic T cells (CD8)

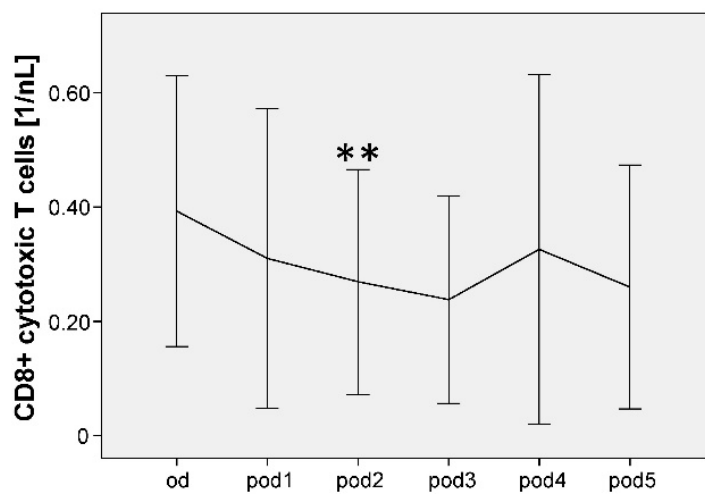

hypothermia must be avoided [5]. Another approach might be postoperative immune stimulation, which was shown to reduce infection days [22]. The impact of this stimulation on cancer metastases and recurrence is unknown and should be further investigated.

B B cells (CD19)

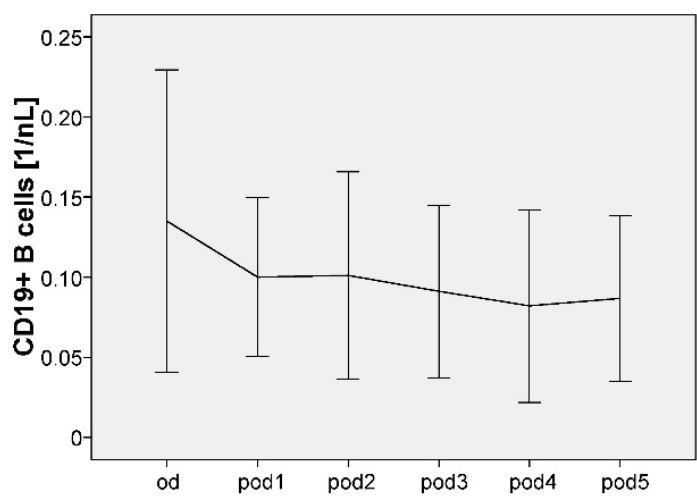

D T helper cells (CD4)

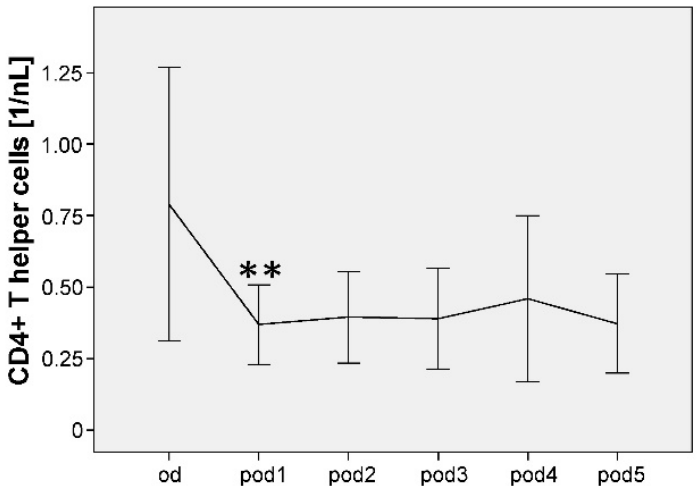

F $\quad$ CD4/CD8 ratio

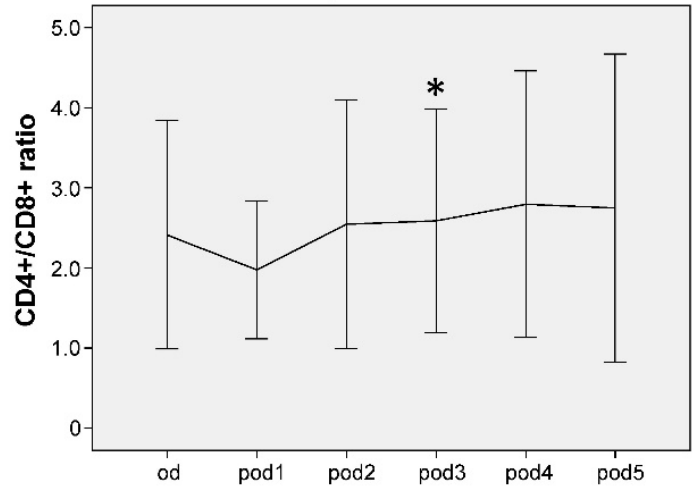

Figure 4. Lymphocytes, B cells, T cells, T helper cells and cytotoxic T cells from day of surgery before surgery (od) until day 5 after surgery (pod5). Lymphocytes decreased on podI, B cells did not show any significant differences. T cells and T helper cells decreased on pod I and cytotoxic T cells decreased on pod2. The ratio of T helper and cytotoxic T cells increased on pod 3 compared to pod I. $* * \mathrm{P}<0.01$, $* \mathrm{P}<0.05$ represent the first significant differences between pre- and postsurgical values. Error bars with $95 \%$ confidence intervals. 
A IFN-y after Con A

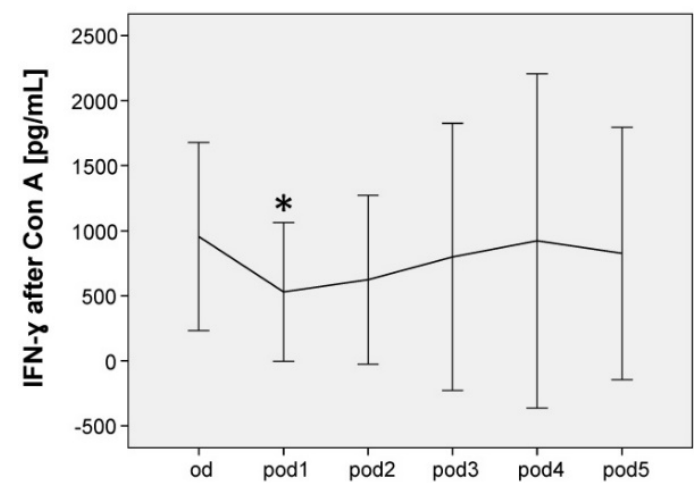

C

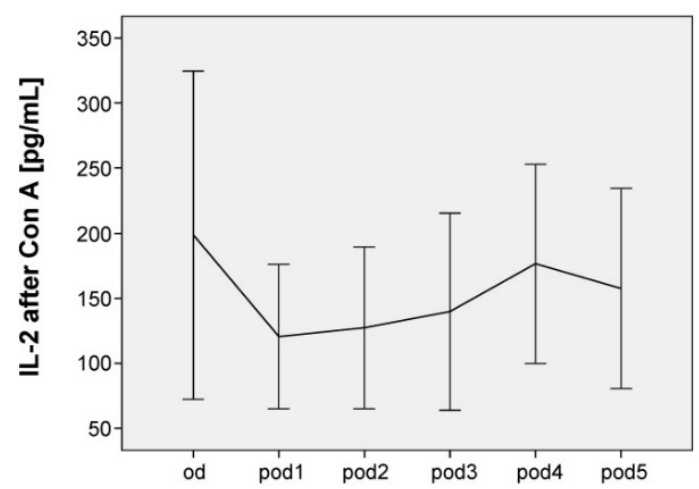

E

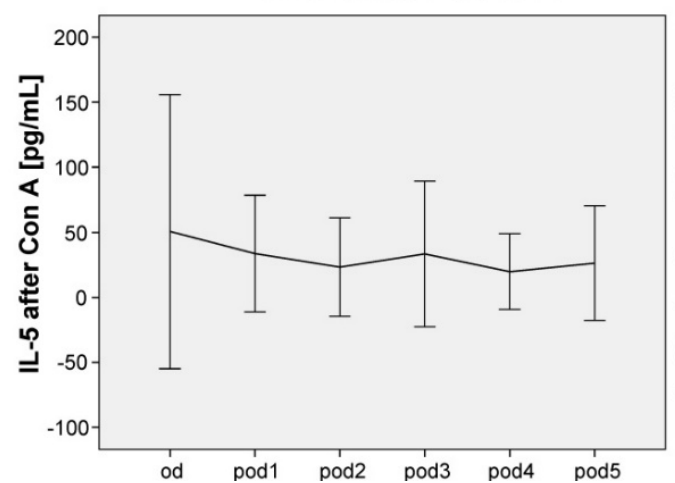

B TNF- $\alpha$ after Con A

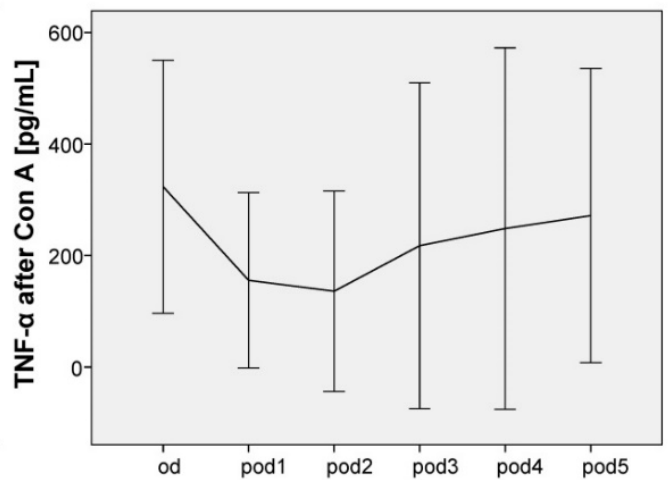

D

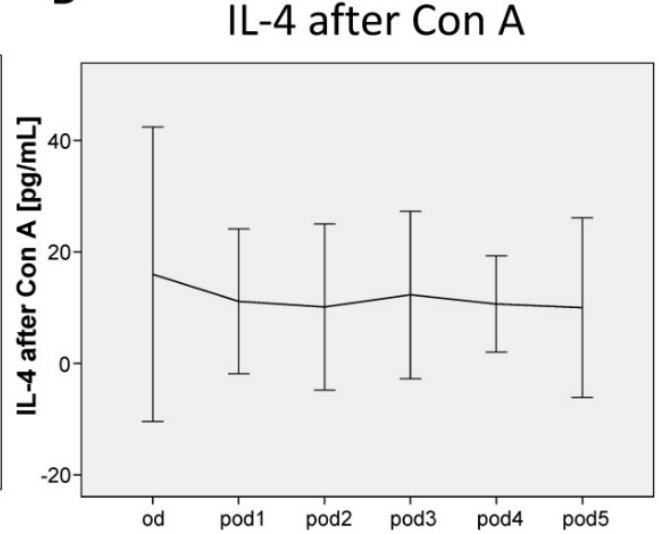

F IL-10 after Con A

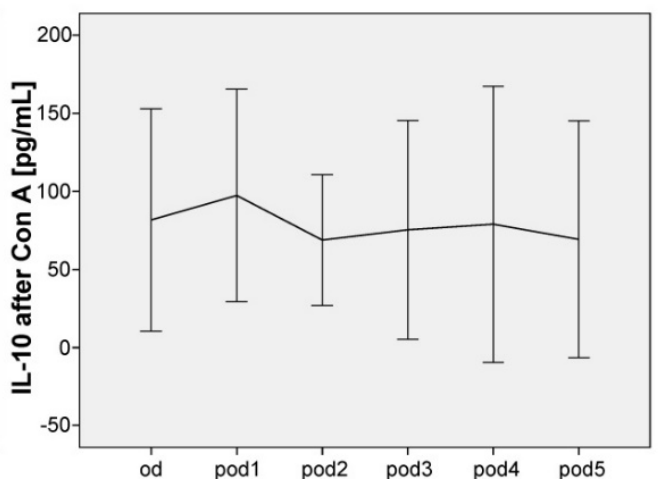

Figure 5. Con A-induced lymphocytic IFN- $y$, TNF- $\alpha$, IL-2, IL-4, IL-5 and IL-10 secretion from day of surgery before surgery (od) until day 5 after surgery (pod5). The Th1 cytokine IFN- $y$ decreased on pod I, TNF- $\alpha$ and IL-2 did not significantly change after surgery. The Th2 cytokines IL-4, IL-5 and IL- 10 did not significantly change after surgery. $*$ P $<0.05$ represents the first significant differences between pre- and postsurgical values. Error bars with $95 \%$ confidence intervals.

This study reveals several limitations. First of all, it is a retrospective subgroup analysis. Secondly, we analyzed only a small sample size of 10 patients, i.e. some results might possibly be not significant. Thirdly, patients were analyzed only until pod5. The course of immune cells and function after this period is unknown. Finally, the optimal threshold level for mHLA-DR $(\leq 10,000 \mathrm{mAb} /$ cell in our study) used to stratify patients with severe surgery-induced immunosuppression is unclear. Studies suggest values between 5,000 and 10,000 $\mathrm{mAb}$ /cell as indicator of severely impaired immune function in critically ill patients $[25,33,34]$.

\section{Conclusions}

Postoperative innate immunity recovered earlier than acquired immunity during severe postoperative immunosuppression. Furthermore, we found a more 
anti- than pro-inflammatory $\mathrm{T}$ cell function on the first day after surgery, while $\mathrm{T}$ cell counts decreased. Further research should focus on strategies to avoid postoperative immune suppression and improve outcome.

\section{Abbreviations}

AMG: German Drug Law

APACHE: Acute Physiology and Chronic Health

Evaluation

ASA: American Society of Anesthesiologists

AUDIT: Alcohol Use Disorders Identification Test

BCRT: Berlin-Brandenburg Center for Regenerative Therapies

Con A: Concanavalin A

EDTA: ethylene diamine tetraacetic acid

FACS: fluorescence-activated cell sorting

ICU: Intensive Care Unit

IL: interleukin

IFN- $\gamma$ : interferon gamma

LaGeSo: Landesamt für Gesundheit und Soziales

Berlin

LPS: lipopolysaccharide

mHLA-DR: human leukocyte antigen DR on monocytes

NK: natural killer cell

od: preoperatively

pod: postoperative day

RCT: randomized controlled trial

SAPS: Simplified Acute Physiology Score

SD: standard deviation

SOFA: Sequential Organ Failure Assessment

Th: T helper cell

TISS: Therapeutic Intervention Scoring System

TNF-a: tumor necrosis factor alpha

Treg: T regulatory cells

\section{Acknowledgements}

We are very grateful to Kathrin Scholtz for monitoring this study, to Anja-Vanessa Philippeit, Dominik Stöber, Julia Schäfer, Carolyn Geipel and Kay Dittrich for data acquisition and help with the database. We thank Victoria Windmann for her help with the manuscript.

The study was performed at the Department of Anesthesiology and Operative Intensive Care Medicine, Campus Charité Mitte and Campus Virchow-Klinikum, Charité - Universitätsmedizin Berlin, Germany.

Clinical trial registered with www.controlledtrials.com (ISRCTN27114642) 05 December 2008.

\section{Authors' contributions}

Conceived and designed the experiments: CS. Performed the experiments: GL, CVH, JK, FY.
Analyzed the data: GL, CVH, JK. Contributed materials / analysis tools: CVH, JK, FY. Wrote the paper: GL, CVH.

\section{Ethics approval and consent to participate}

This clinical trial was approved by the Ethics Committee of the Landesamt für Gesundheit und Soziales Berlin (LaGeSo), Germany (ref ZSEK15287/08) on September 01, 2008. The study further meets the requirements set out by the ICH-GCP, Declaration of Helsinki and the German Drug Law (AMG). Written informed consent was obtained from the patients.

\section{Availability of data and materials}

Due to legal restrictions imposed by the Ethics Committee of the Landesamt für Gesundheit und Soziales Berlin (LaGeSo) and the data protection commissioner of the Charité, public sharing of study data with other researchers or entities is not allowed. Requests may be sent to dai-researchdata@charite.de.

\section{Funding}

Deutsche Forschungsgemeinschaft (DFG SP432-1, http://www.dfg.de/, the funders had no role in study design, data collection and analysis, decision to publish, or preparation of the manuscript), Charité - Universitätsmedizin Berlin (www.charite.de, the funders had no role in study design, data collection and analysis, decision to publish, or preparation of the manuscript).

\section{Competing Interests}

The authors have declared that no competing interest exists.

\section{References}

1. Bartal I, Melamed R, Greenfeld K, Atzil S, Glasner A, Domankevich V, et al. Immune perturbations in patients along the perioperative period: alterations in cell surface markers and leukocyte subtypes before and after surgery. Brain, behavior, and immunity. 2010; 24: 376-86

2. Goldfarb Y, Sorski L, Benish M, Levi B, Melamed R, Ben-Eliyahu S. Improving postoperative immune status and resistance to cancer metastasis: a combined perioperative approach of immunostimulation and prevention of excessive surgical stress responses. Annals of surgery. 2011; 253: 798-810.

3. Costa A, Benedetto V, Ricci C, Merlin P, Borelli P, Fadda E, et al. Endocrine, hematological and immunological changes in surgical patients undergoing general anesthesia. The Italian journal of surgical sciences / sponsored by Societa italiana di chirurgia. 1989; 19: 41-9.

4. Kendall SJ, Weir J, Aspinall R, Henderson D, Rosson J. Erythrocyte transfusion causes immunosuppression after total hip replacement. Clinical orthopaedics and related research. 2000: 145-55.

5. Beilin B, Shavit Y, Razumovsky J, Wolloch Y, Zeidel A, Bessler H. Effects of mild perioperative hypothermia on cellular immune responses. Anesthesiology, 1998; 89: 1133-40.

6. Pirbudak Cocelli L, Ugur MG, Karadasli H. Comparison of effects of low-flow sevoflurane and desflurane anesthesia on neutrophil and T-cell populations. Curr Ther Res Clin Exp. 2012; 73: 41-51.

7. Wakefield CH, Carey PD, Foulds S, Monson JR, Guillou PJ. Changes in major histocompatibility complex class II expression in monocytes and T cells of patients developing infection after surgery. The British journal of surgery. 1993; 80: 205-9.

8. Shakhar G, Ben-Eliyahu S. Potential prophylactic measures against postoperative immunosuppression: could they reduce recurrence rates in oncological patients? Ann Surg Oncol. 2003; 10: 972-92. 
9. Tartter PI, Steinberg B, Barron DM, Martinelli G. The prognostic significance of natural killer cytotoxicity in patients with colorectal cancer. Archives of surgery. 1987; 122: 1264-8.

10. Greenfeld K, Avraham R, Benish M, Goldfarb Y, Rosenne E, Shapira Y, et al Immune suppression while awaiting surgery and following it: dissociations between plasma cytokine levels, their induced production, and NK cell cytotoxicity. Brain, behavior, and immunity. 2007; 21: 503-13.

11. Koerner P, Westerholt A, Kessler W, Traeger T, Maier S, Heidecke CD. [Surgical trauma and postoperative immunosuppression]. Der Chirurg; Zeitschrift fur alle Gebiete der operativen Medizen. 2008; 79: 290-4.

12. Veenhof AA, Sietses C, von Blomberg BM, van Hoogstraten IM, vd Pas MH, Meijerink WJ, et al. The surgical stress response and postoperative immune function after laparoscopic or conventional total mesorectal excision in rectal cancer: a randomized trial. International journal of colorectal disease. 2011; 26: 53-9.

13. Ogawa K, Hirai M, Katsube T, Murayama M, Hamaguchi K, Shimakawa T, et al. Suppression of cellular immunity by surgical stress. Surgery. 2000; 127: 329-36.

14. Cristaldi M, Rovati M, Elli M, Gerlinzani S, Lesma A, Balzarotti L, et al. Lymphocytic subpopulation changes after open and laparoscopic cholecystectomy: a prospective and comparative study on 38 patients. Surgical laparoscopy \& endoscopy. 1997; 7: 255-61.

15. Shafir M, Bekesi JG, Papatestas A, Slater G, Aufses AH, Jr. Preoperative and postoperative immunological evaluation of patients with colorectal cancer. Cancer. 1980; 46: 700-5.

16. Brune IB, Wilke W, Hensler T, Holzmann B, Siewert JR. Downregulation of T helper type 1 immune response and altered pro-inflammatory and anti-inflammatory $\mathrm{T}$ cell cytokine balance following conventional but not laparoscopic surgery. Am J Surg. 1999; 177: 55-60.

17. Buggy DJ, Smith G. Epidural anaesthesia and analgesia: better outcome after major surgery?. Growing evidence suggests so. Bmj. 1999; 319: 530-1.

18. Ditschkowski M, Kreuzfelder E, Rebmann V, Ferencik S, Majetschak M, Schmid EN, et al. HLA-DR expression and soluble HLA-DR levels in septic patients after trauma. Ann Surg. 1999; 229: 246-54.

19. Hensler $T$, Hecker $H$, Heeg $K$, Heidecke CD, Bartels $H$, Barthlen W, et al. Distinct mechanisms of immunosuppression as a consequence of major surgery. Infection and immunity. 1997; 65: 2283-91.

20. Franke A, Lante W, Kurig E, Zoller LG, Weinhold C, Markewitz A. Hyporesponsiveness of $\mathrm{T}$ cell subsets after cardiac surgery: a product of altered cell function or merely a result of absolute cell count changes in peripheral blood? European journal of cardio-thoracic surgery : official journal of the European Association for Cardio-thoracic Surgery. 2006; 30: 64-71.

21. Smith JW, Gamelli RL, Jones SB, Shankar R. Immunologic responses to critical injury and sepsis. Journal of intensive care medicine. 2006; 21: 160-72.

22. Spies C, Luetz A, Lachmann G, Renius M, von Haefen C, Wernecke KD, et al. Influence of Granulocyte-Macrophage Colony-Stimulating Factor or Influenza Vaccination on HLA-DR, Infection and Delirium Days in Immunosuppressed Surgical Patients: Double Blind, Randomised Controlled Trial. PloS one. 2015; 10: e0144003.

23. Lachmann G, Kurth J, von Haefen C, Yuerek F, Wernecke KD, Spies C. In vivo application of Granulocyte-Macrophage Colony-stimulating Factor enhances postoperative qualitative monocytic function. Int J Med Sci. 2017; 14: 367-75.

24. Spies C, Kox W, Kastrup M, Melzer-Gartzke C. SOPs in Intensivmedizin und Notfallmedizin: Alle relevanten Standards und Techniken für die Klinik. 2013: Stuttgart, Georg Thieme Verlag; 2013.

25. Docke WD, Hoflich C, Davis KA, Rottgers K, Meisel C, Kiefer P, et al. Monitoring temporary immunodepression by flow cytometric measurement of monocytic HLA-DR expression: a multicenter standardized study. Clin Chem. 2005; 51: 2341-7.

26. Hansen S, Baptiste KE, Fjeldborg J, Horohov DW. A review of the equine age-related changes in the immune system: comparisons between human and equine aging, with focus on lung-specific immune-aging. Ageing research reviews. 2015; 20: 11-23.

27. Albertsmeier M, Quaiser D, von Dossow-Hanfstingl V, Winter H, Faist E, Angele MK. Major surgical trauma differentially affects T-cells and APC. Innate immunity. 2015; 21: 55-64.

28. Oppenheim JJ, Yang D. Alarmins: chemotactic activators of immune responses. Current opinion in immunology. 2005; 17: 359-65.

29. Munford RS, Pugin J. Normal responses to injury prevent systemic inflammation and can be immunosuppressive. American journal of respiratory and critical care medicine. 2001; 163: 316-21.

30. Delogu G, Moretti S, Antonucci A, Marcellini S, Masciangelo R, Famularo G, et al. Apoptosis and surgical trauma: dysregulated expression of death and survival factors on peripheral lymphocytes. Archives of surgery. 2000; 135: 1141-7.

31. Tatsumi H, Ura H, Ikeda S, Yamaguchi K, Katsuramaki T, Asai Y, et al. Surgical influence on $\mathrm{TH} 1 / \mathrm{TH} 2$ balance and monocyte surface antigen expression and its relation to infectious complications. World journal of surgery. 2003; 27: 522-8.

32. Ahlers O, Nachtigall I, Lenze J, Goldmann A, Schulte E, Hohne C, et al. Intraoperative thoracic epidural anaesthesia attenuates stress-induced immunosuppression in patients undergoing major abdominal surgery. $\mathrm{Br} \mathrm{J}$ Anaesth. 2008; 101: 781-7.

33. Meisel C, Schefold JC, Pschowski R, Baumann T, Hetzger K, Gregor J, et al. Granulocyte-macrophage colony-stimulating factor to reverse sepsis-associated immunosuppression: a double-blind, randomized, placebo-controlled multicenter trial. American journal of respiratory and critical care medicine. 2009; 180: 640-8.

34. Cheron A, Floccard B, Allaouchiche B, Guignant C, Poitevin F, Malcus C, et al. Lack of recovery in monocyte human leukocyte antigen-DR expression is independently associated with the development of sepsis after major trauma. Crit Care. 2010; 14: R208. 